\title{
THICK FILM CIRCUITS FOR TELECOMMUNICATIONS EQUIPMENT
}

\author{
WILLIAM B. GRUPEN and HOWARD M. COHEN \\ Bell Telephone Laboratories, Incorporated, 555 Union Boulevard, Allentown, Pennsylvania 18103
}

\begin{abstract}
The use of thick film passive networks and hybrid integrated circuits by the Bell System is described in this paper. Included is a discussion of how attributes of thick films such as a large resistance range, high power handling capability, a bipolar capacitor and multilevel interconnection capability are used to provide a range of circuit properties that supplement the properties available in a well-established thin film technology. Design aids permitting a rapid transition from an electrical schematic to a finished circuit, special laser trimming procedures and material blending procedures are described for resistor networks. Also included are assembly and packaging techniques suitable for high reliability and low cost manufacturing. Several hybrid integrated circuits which benefit from the use of thick films are described in terms of their particular electrical or structural requirements. Included is a family of power converter circuits illustrating the advantages of a modular approach to circuit design in which functional resistor trimming provides a number of different circuit characteristics. Several applications involving the use of thick films in combination with thin films to provide special hybrid circuit properties not available from thin films alone are described. These and other possible uses of the two technologies in the same circuit are discussed in terms of combining the best properties of each technology to provide higher reliability and lower circuit cost. Finally, the future for the use of thick films in the Bell System is discussed.
\end{abstract}

\section{INTRODUCTION}

The Bell System use of thick films in hybrid and passive circuits began in 1974. Forecasts indicate that about $10 \%$ of the circuits manufactured by Western Electric in 1982 will contain thick films alone and that an additional $30 \%$ will involve the use of thin and thick films together on one substrate. The balance will contain thin films alone.

The use of the thick film technology as an adjunct to a well-established thin film technology was prompted by a number of significant improvements in materials and equipment made during the ten-year period prior to 1974. The combined effects of these improvements resulted in a film technology with the following characteristics which were considered to be beneficial to a number of Bell System applications.

1) A wide range in sheet resistance permitting the design of medium tolerance resistors from $10 \Omega$ to 100 megohms.

2) A high power handling capability permitting circuit operating temperatures up to $300^{\circ} \mathrm{C}$.

3) A bipolar capacitor capable of operating above 50 volts and at high frequencies.

4) The ability of film circuit components to withstand assembly and packaging operations involving temperatures up to $500^{\circ} \mathrm{C}$.

5) A multilevel interconnection capability.

6) Low cost manufacturing equipment.

7) Acceptable reliability.

An examination of these characteristics in the context of those already available in the thin film technology led to the conclusion that the wide resistance range, high temperature operation and multilevel interconnection capabilities should be considered as a means of extending our overall hybrid circuit design capabilities. This has resulted in the initial use of thick films in four areas: resistor networks, special components in thin film circuits, power supply circuits and high voltage circuits which will be described in more detail below. Before proceeding with this, it is important to note two decisions that affected the manner in which resources were allocated to development activities.

The first decision was to make use of commercial pastes, which were available from a number of outside suppliers. This did require that a paste characterization effort be included in development activities, but it was considerably smaller than the effort that would have been required to develop an in-house family of resistor, 
dielectric and conductor pastes. The second decision was to fabricate circuits in as large a batch as possible rather than the one at a time technique which requires high speed facilities and rather intricate magazine handling procedures. This decision made it important in an economic sense to include as many circuits as possible on the selected substrate and necessitated the inclusion of work on circuit density improvements in development activities.

\section{RESISTOR NETWORKS}

The primary driving force in the use of resistor networks is printed wiring board space savings. It is frequently found that a resistor network in a dual in-line pack format occupies less than one-half the board space required by an equivalent array of discrete resistors. Secondary reasons for the use of networks include lower overall component insertion cost and in some cases improved electrical behavior for the same or lower component cost. In this context, resistor networks are being designed into most new customer premises, transmission and switching systems.

The design of a resistor network begins with the experimental acquisition of data on the effects of resistor length on sheet resistance. These effects are the result of nonuniformities in resistor cross section and diffusion of the termination material into the resistor. Typical data are shown in Figure 1. These data are normallized with respect to the nominal sheet resistance values. It should be noted that when resistor printing is accomplished properly, sheet resistance does not depend on resistor width.

Most suppliers of thick film pastes provide them in decade values of sheet resistance. It is often desirable, in order to reduce circuit area, to use an intermediate value of sheet resistance. To make this possible, suppliers generally design their pastes so that adjacent members of a given family may be blended. The exact quantities of these adjacent members required to obtain a desired sheet resistance must be obtained empirically. An example of a blending curve for the DuPont 1431 and 1441 pastes is shown in Figure 2.

Data such as that shown in Figures 1 and 2 are used with an interactive computer program which permits the circuit designer to determine resistor length, width and paste composition. The program accounts for the effects of termination material and variations between paste suppliers. Its output is a deck of punched cards describing the resistor geometry in a format suitable for entry into an interactive circuit design system which is used for the layout of both resistor networks and hybrid circuits.

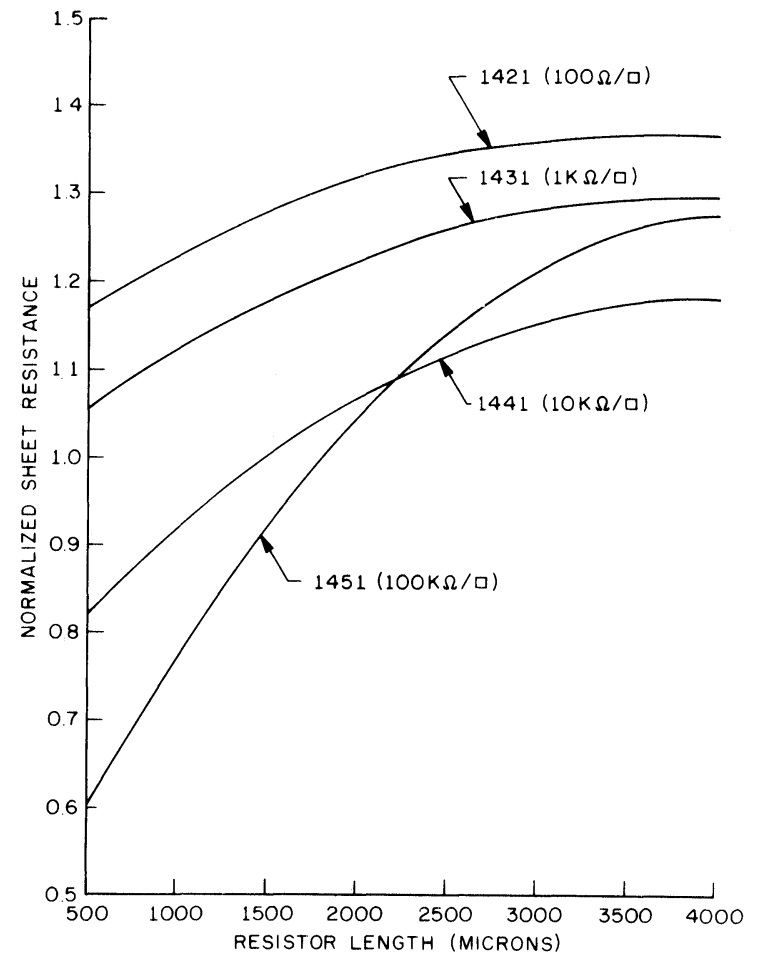

FIGURE 1 Normalized resistance vs. resistor length for some Dupont 1400 series resistor materials.

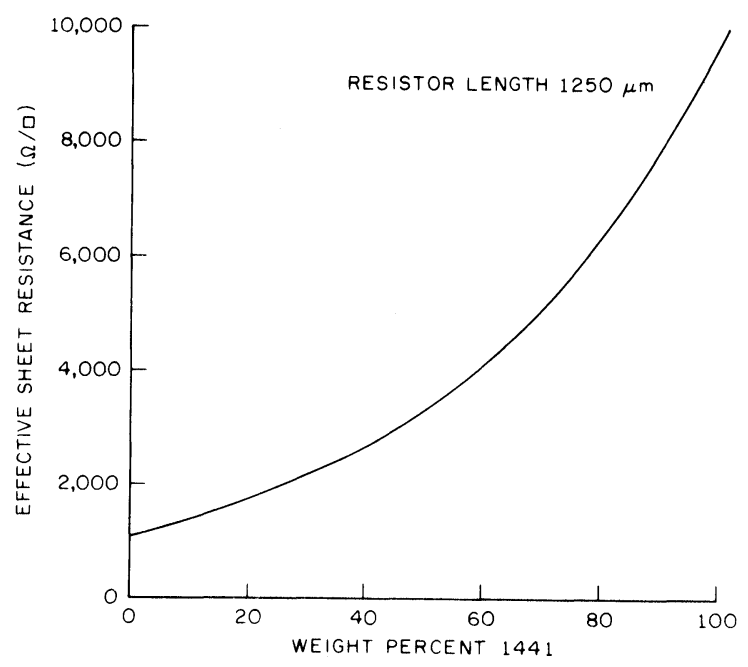

FIGURE 2 Blending curve for Dupont 1431 and 1441 resistor pastes.

Following the layout of a resistor network, conductors and resistors are fabricated in a batch format on a 3.75 in. by 4.5 in. $96 \%$ alumina substrate using standard printing and firing procedures. The result is shown in Figure 3. An overglaze is added next 


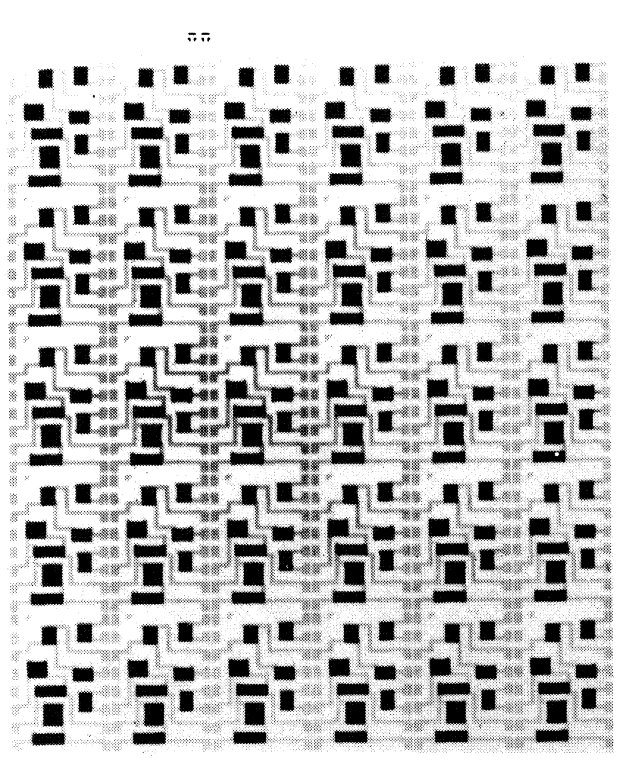

:;;

FIGURE 3 A 600 mil wide resistor network replicated 30 times on a 3.75 in. $\times 4.5$ in. $96 \%$ alumina ceramic.

to inhibit silver migration between palladium-silver conductors in high humidity environments. Following this, the resistors are laser trimmed and a silicone based covercoat is applied to provide mechanical protection during the subsequent assembly operations. The substrate is then separated into individual circuits and external clip leads are attached by soldering. The resulting circuit, shown in the upper part of Figure 4, constitutes a finished ceramic dual in-line pack (DIP). A ceramic cap which is attached by epoxy to one side of the circuit is required if the circuit is to be inserted into a printed wiring board by automatic techniques. Such a package is shown in the lower part of Figure 4.

Two ceramic DIP's having $300 \mathrm{mil}$ and $600 \mathrm{mil}$ widths are presently being manufactured. The $300 \mathrm{mil}$ ceramic DIP will be replaced this year by a plastic DIP which is expected to be less costly and more rugged. A considerable effort was necessary to retain a $0.5 \%$ end-of-life tolerance that is typical of the ceramic DIP. This has resulted in the development of techniques for minimizing forces exerted on the strain sensitive resistors by the plastic encapsulant.

The plastic DIP contains a 200 mil wide ceramic. Eighty such ceramics are placed on one $3.75 \times 4.5$ in. master substrate. A printing rate of 500 substrate prints per hour is achieved with this substrate. This is equivalent to 40,000 circuit prints per hour, which translates to less than one cent per circuit print.

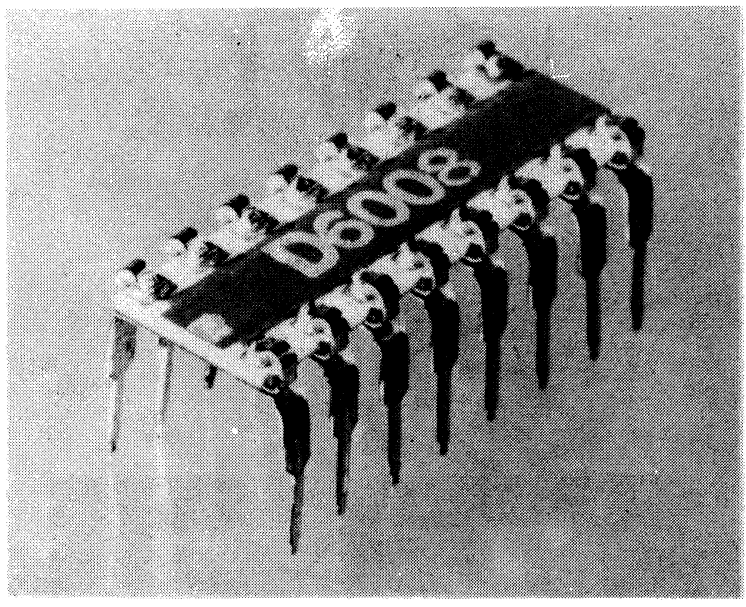

\section{CERAMIC PACKAGE}

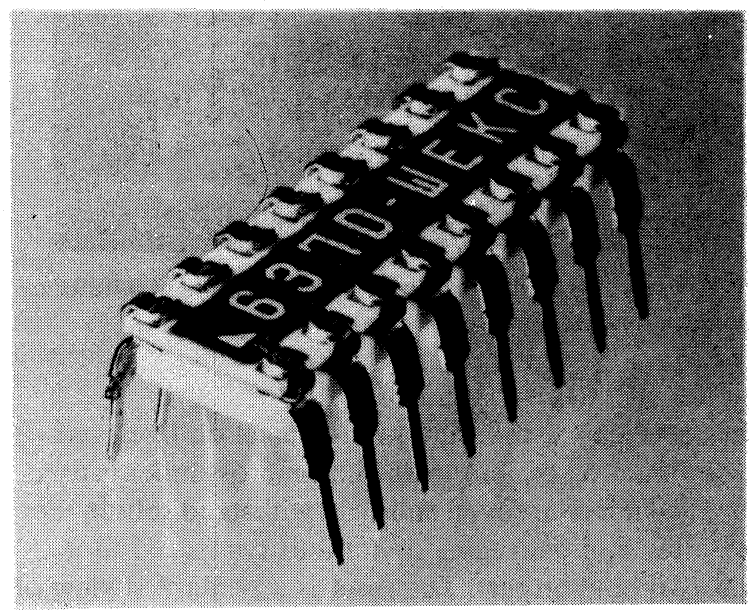

CERAMIC PACKAGE WITH CERAMIC CAP ON BOTTON FOR MACHINE INSERTION CAPABILITY

FIGURE 4 Ceramic dual in-line package.

\section{HYBRID INTEGRATED CIRCUITS}

The primary function of thick film integrated circuits is to provide features that are difficult or impossible to obtain economically in the discrete device-printed wiring board format or the thin film hybrid integrated circuit-printed wiring board format. On this basis, three areas were elected for the application of thick films.

The first area of application involves the use of thick film components in thin film hybrid circuits where special properties not available in thin films are required. Figure 5 shows one such application. The circuit contains active devices, thin film tantalum 


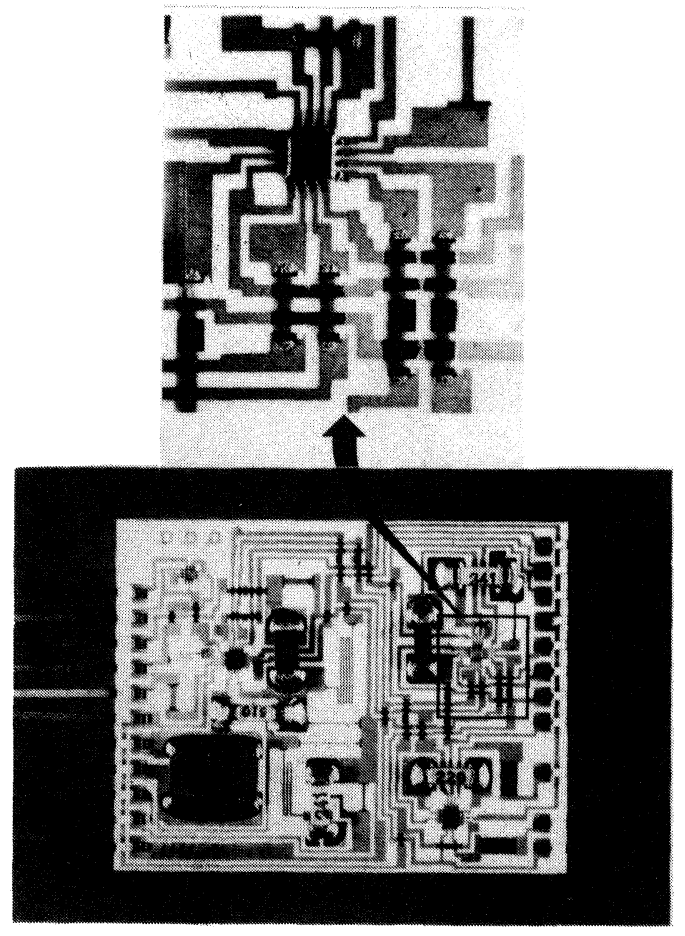

FIGURE 5 A thin film hybrid integrated circuit with thick film crossunders. (Substrate size $4.5 \mathrm{~cm} \times 3.5 \mathrm{~cm}$.)

nitride resistors, thin film interconnection lines and thick film crossunders. A thick film crossunder is used because of its improved ability to withstand subsequent assembly and packaging operations compared to its thin film counterpart. The inset is an enlarged view of several of these crossunders. A crossunder is fabricated by first screen printing and firing a 5 mil wide gold stripe and then printing and firing an overlaying dielectric patch. This dielectric must be able to withstand the etchants used in the thin film processes which are used to complete the circuit. As in the case of all hybrid circuits, the final fabrication step is the application of a room temperature vulcanizing silicone rubber for environmental protection.

Figure 6 shows a second application of thick and thin films on one circuit. In this case the circuit contains several active devices, thin film tantalum nitride resistors, thin film interconnection lines and two appliqued thin film resistor networks. The resistor networks contain resistors ranging from $200 \mathrm{~K} \Omega$ to $10 \mathrm{M} \Omega$ which would require an unacceptably large amount of substrate area if they were fabricated using thin film tantalum nitride. The resistor networks are attached to the primary circuit using a solder bump technique.

The second area in which thick films have been used

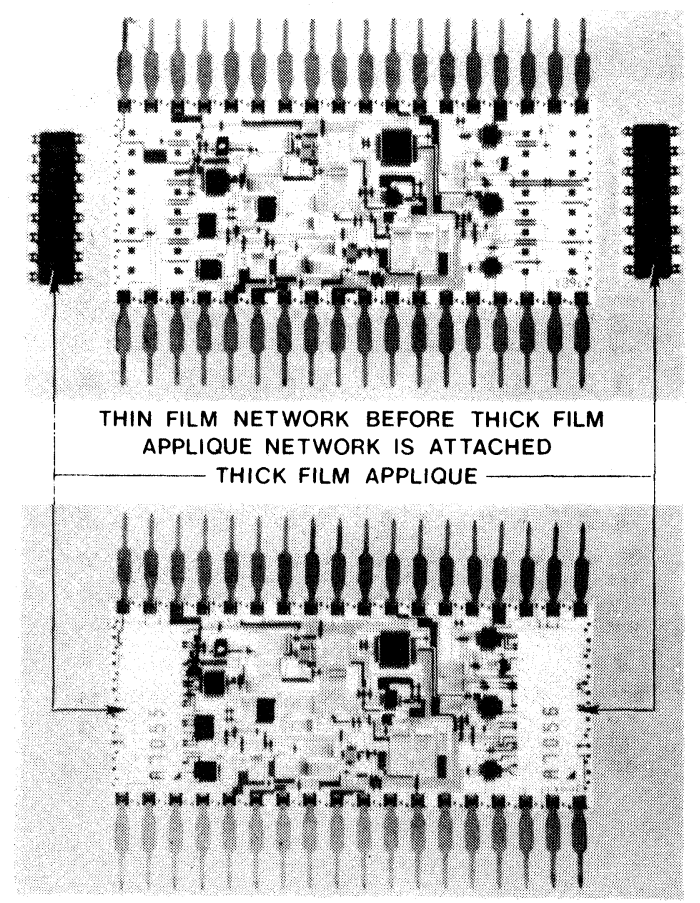

FIGURE 6 Thin film hybrid integrated circuit with appliqued thick film resistor networks. (Substrate size $4.5 \mathrm{~cm} \times 2 \mathrm{~cm}$.)

in hybrid circuits involves high voltage applications where discrete silicon devices are interconnected by eutectic die bonding and wire bonding procedures. The upper part of Figure 7 shows an example of such a circuit which contains eight discrete diodes and eight thick film resistors on the top of the circuit. There are four additional resistors on the bottom of the circuit. In this application, the ability of thick film components to withstand the series of high temperature operations associated with external lead and die attachment has been taken advantage of. The finished circuit, protected with a screened epoxy covercoat, is shown in the lower part of the figure.

The third area in which thick films are used is the replacement of discrete component circuits in power supplies having up to about 100 watts output by thick film hybrid circuits. Here the high power handling (high temperature operation) capabilities of thick films are important. Figure 8 shows a control circuit used in a family of power supplies. It contains three beam leaded silicon integrated circuits, forty-four resistors and four barium titanate chip capacitors. A feature of this circuit design is an active laser trimming operation that is used to adjust several critical resistors after the silicon integrated circuits are attached. In this manner one circuit layout provides a family of converters with outputs from 5 to 30 volts. 


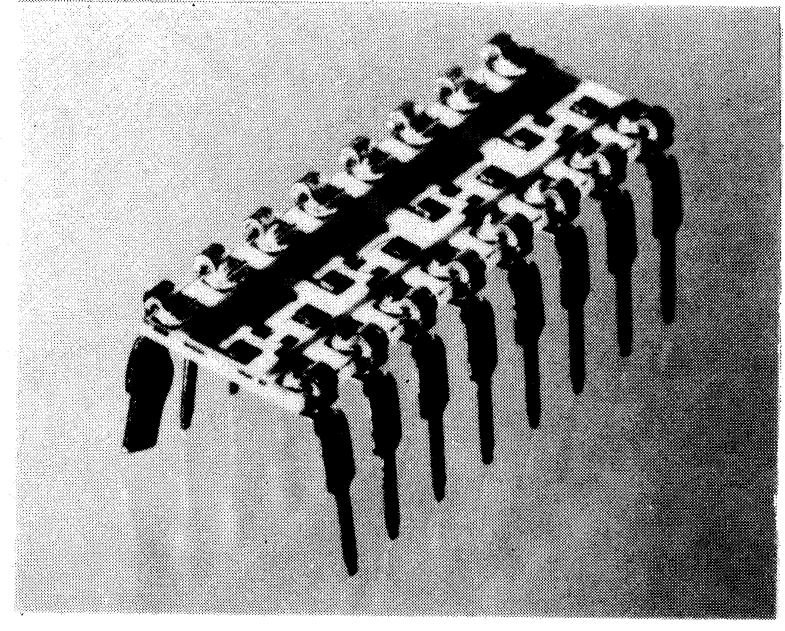

\section{UNENCAPSULATED THICK FILM CIRCUIT WITH HIGH VOLTAGE DIODES}

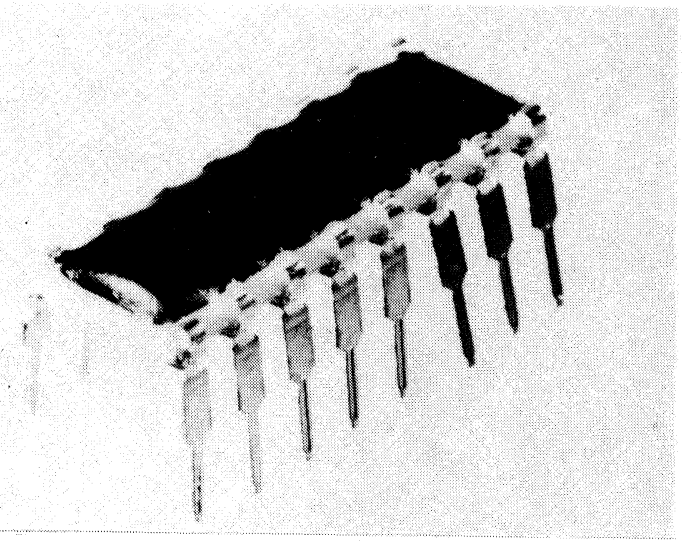

ENCAPSULATED CIRCUIT

FIGURE 7 Thick film circuit with discrete diodes used in a high voltage application.

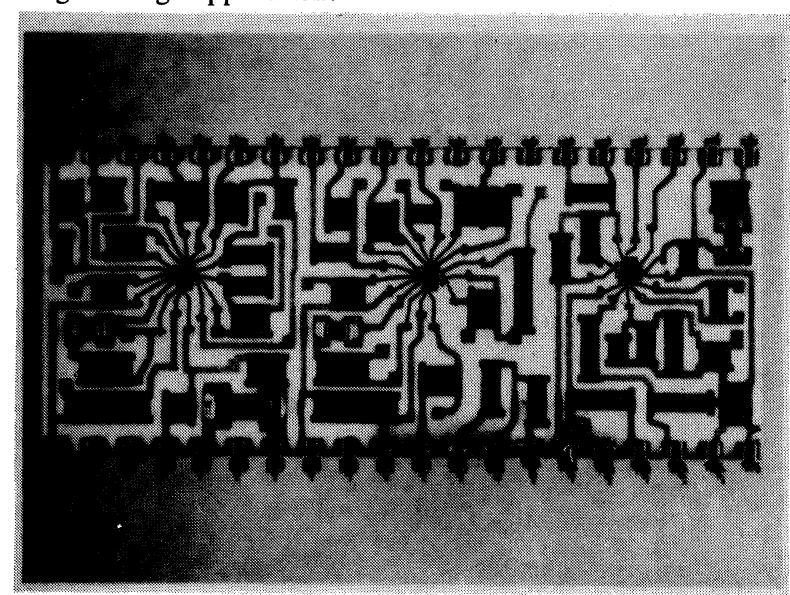

FIGURE 8 Thick film power control circuit. (Substrate size $5 \mathrm{~cm} \times 2 \mathrm{~cm}$.)

\section{THE FUTURE}

Thick films will continue to be used by the Bell System in the three areas discussed above: resistor networks, combined thin and thick film hybrids and special hybrids. It is expected that the areas having the most potential for further expansion are the combined film hybrids and special hybrids. As an example of this, Figure 9 shows a microcomputer circuit that is currently under study. It contains three ceramic chip carriers on a three interconnection level thick film substrate. The chip carrier on the right contains four input-output beam leaded bipolar devices on a small thin film substrate. The chip carrier in the center contains a CMOS microprocessor chip and the chip carrier on the left contains an MOS memory chip. This packaging technique involving the use of chip carriers, the high resolution capabilities of thin films and the multilevel capabilities of thick films can accommodate a variety of different device structures and device attachment procedures.

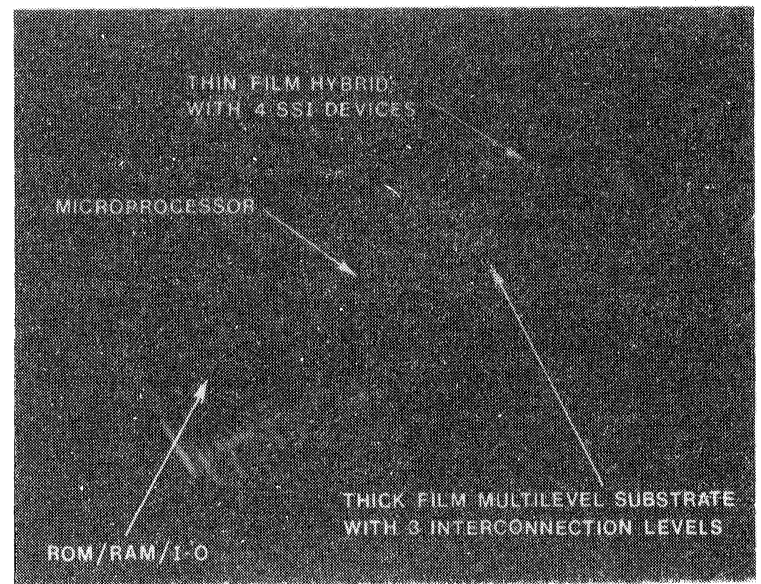

FIGURE 9 An experimental microcomputer circuit. (Substrate size $6.25 \mathrm{~cm} \times 2.25 \mathrm{~cm}$.) 

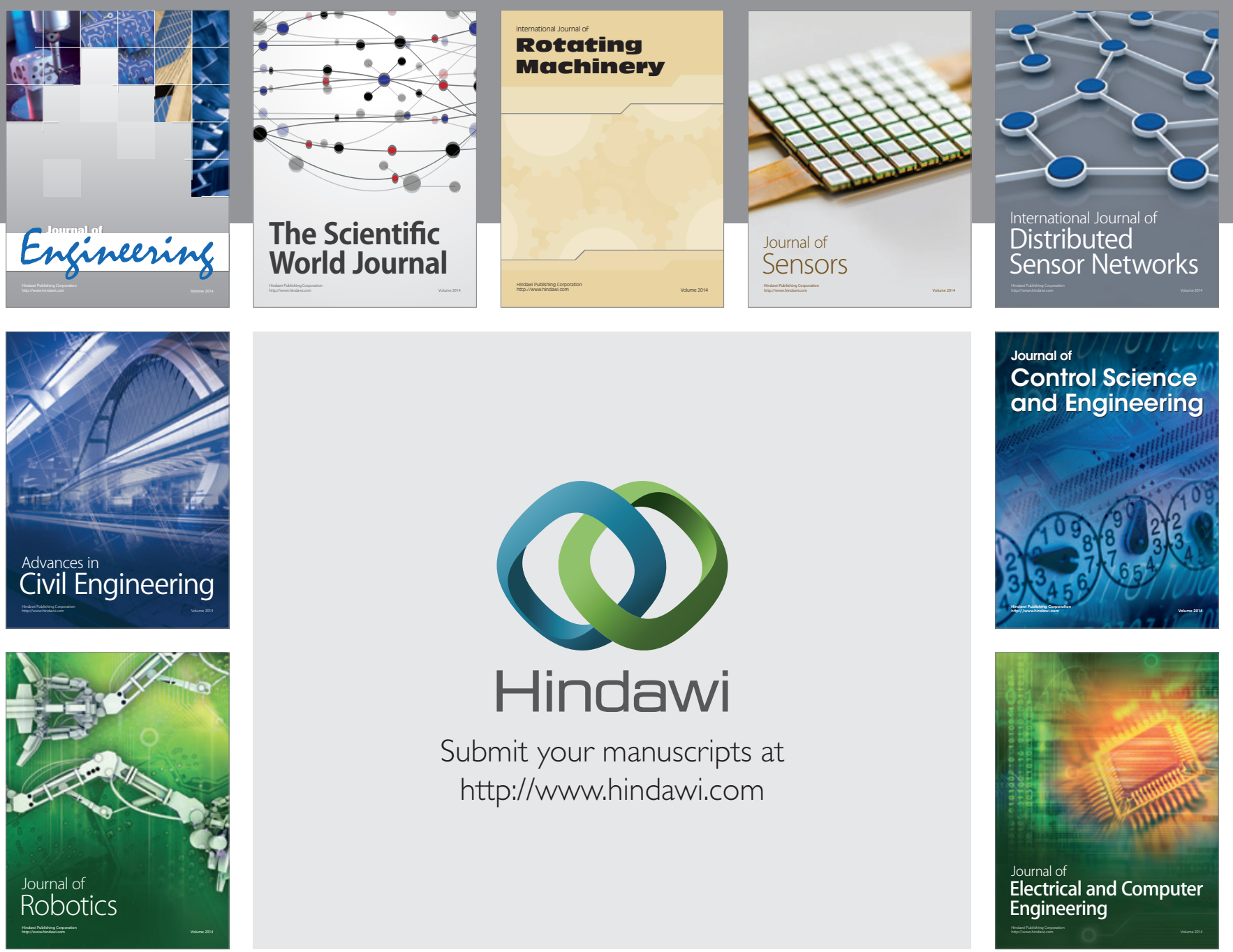

Submit your manuscripts at

http://www.hindawi.com
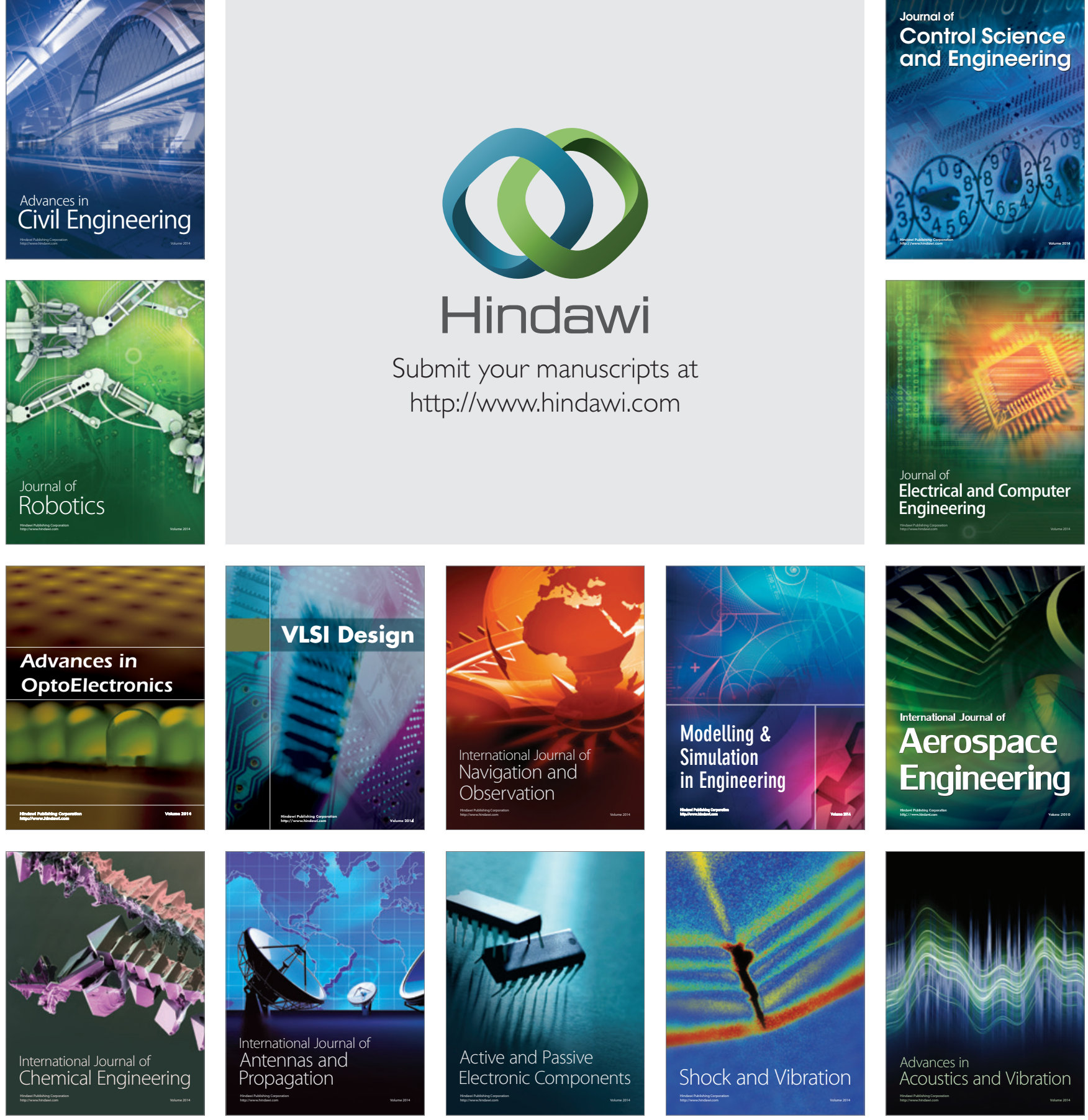